\title{
Origines de la musique en l'homme, selon Pascal Quignard
}

Joseph Brami

\section{(2) OpenEdition}

\section{Journals}

Édition électronique

URL : http://journals.openedition.org/rief/827

DOI : $10.4000 /$ rief. 827

ISSN : 2240-7456

\section{Éditeur}

Seminario di filologia francese

Référence électronique

Joseph Brami, «Origines de la musique en l'homme, selon Pascal Quignard », Revue italienne d'études françaises [En ligne], 2 | 2012, mis en ligne le 15 décembre 2012, consulté le 01 mai 2019. URL : http:// journals.openedition.org/rief/827 ; DOI : 10.4000/rief.827

Ce document a été généré automatiquement le 1 mai 2019.

\section{(c) (i) (9)}

Les contenus de la RIEF sont mis à disposition selon les termes de la Licence Creative Commons Attribution - Pas d'Utilisation Commerciale - Pas de Modification 4.0 International. 


\title{
Origines de la musique en l'homme, selon Pascal Quignard
}

\author{
Joseph Brami
}

1 Parmi les écrivains français contemporains, Quignard est peut-être celui dont l'œuvre se caractérise le plus par sa constante référence à la musique. Pensons à Tous les matins $d u$ monde, qui est peut-être son roman le plus connu, consacré à l'histoire complexe et tumultueuse de deux compositeurs et violistes baroques, Marin Marais et Sainte Colombe ${ }^{1}$ . Ce roman n'est pourtant que la partie visible de l'iceberg: les livres de Quignard sont tous peuplés de musiciens. Mais le discours sur la musique ne passe pas nécessairement à travers eux. Prenant souvent la parole à la première personne, Quignard peut évoquer tant la place de la musique dans sa famille que dans son expérience personnelle, se constituant ainsi lui-même comme personnage dans son œuvre, figure d'auteur, persona littéraire. Or, évoquant cette persona, il faut en préciser une caractéristique : Quignard laisse souvent passer un jeu de dédoublements et d'échos entre elle et ses personnages de fictions. Et, par ce jeu, à travers cette persona, ce Je quignardien, le discours sur la musique rayonne dans l'ensemble de l'œuvre.

Quignard-persona accomplit un travail d'écriture et de pensée en avançant sur le mode de l'autoportrait. Pour lui, parler de la musique implique parler de lui-même; mais également de l'homme en général. De fait, son but est de dégager une anthropologie - des données pour une connaissance de l'homme; et une éthique - une morale de comportement. Par ailleurs, il faut ajouter que si sa réflexion semble, en son arrière-plan, nourrie jusqu'à un certain point de lectures psychanalytiques, il maintient néanmoins son discours dans l'ordre du poétique. On peut dire que là où le discours analytique aide à trouver les mots pour se dire, le discours quignardien sur la musique tient le rôle du creusement métaphorique de soi que le sujet opère dans une analyse. Étudier ce discours, c'est suivre Quignard dans un registre de métaphores et comprendre en quoi la musique est pour lui le lieu poétique de sa singularité d'homme et d'écrivain, le mythe fondateur de sa scène fantasmatique de l'écriture.

3 La réflexion de Quignard prend place dans le cadre des discours esthétiques et littéraires des $\mathrm{XX}^{e}$ et $\mathrm{XXI}^{\mathrm{e}}$ siècles qui disent le rongement permanent du monde et qui développent 
une critique de tout idéalisme. La musique, chez lui, est langage des « ombres errantes » $\mathrm{du}$ «dernier royaume». Elle se fonde sur un rapport constant à la mort, sans la conscience duquel l'homme nierait la part de vérité sombre qu'elle dit sur l'être humain.

4 Platon a écrit que la musique donne une âme à nos cœurs et des ailes à la pensée. C'est un des lieux communs les plus universels: Quignard ne le partage pas. Il pense que la musique « fait mal » $(H M, 218)^{2}$ et « dit le mal », et il développe cette idée dans La Leçon de la musique et La Haine de la musique. En réalité, la « leçon » ne va pas sans « haine », sans rapport à la mort surtout; la "haine » ne va pas sans «leçon", c'est-à-dire sans conscience de ce qu'est la musique et de ce qu'elle dit de l'homme, de son rapport au monde.

5 Le paradoxe quignardien du discours sur la musique part d'une expérience existentielle vécue par tout adolescent mâle : la mue. Quignard voit en elle un véritable traumatisme puisqu'il s'agit d'une expérience qui, d'après lui, explique tout : la musique, bien sûr et, à travers elle, la condition tragique de l'être humain.

6 Dans Vie secrète, on lit: « Ma voix est sourde. Rien n'a jamais pu la poser depuis une mue désastreuse qui me fit être rejeté des deux chorales qui faisaient ma joie. Mue qui me bannit à jamais non seulement de tous les chants mais même de tous les fredonnements » (VS, 71). Et, dans La Haine de la musique : «Enfant, je chantai. Adolescent, comme tous les adolescents, ma voix se brisa. Mais elle demeura étouffée et perdue» (HM, 154). Une double conséquence naît de cette brisure, l'une formulée en termes existentiels : à l'évocation de cette expérience, Quignard déclare : «je m'ensevelis [alors] passionnément dans la musique instrumentale» (154) - notons la connotation funèbre du verbe « s'ensevelir » pour parler de son refuge dans la musique. Deuxième conséquence : « Il y a un lien direct entre la musique et la mue » (154). La conviction de l'existence de ce lien est à la base du discours sur la musique.

7 "Je traite de la mue », dit le Je dès la troisième phrase d'« Un épisode de la vie de Marin Marais », premier texte de La Leçon de musique (LM, 11). Cet « épisode » offre un exemple des dédoublements entre personnages et persona. Selon le récit qui en est donné, Marin Marais est passé, au XVII ${ }^{\mathrm{e}}$ siècle, par la même expérience que le Je quignardien au XXe ${ }^{\mathrm{e}}$. Enfant de chœur dans la maîtrise de Saint-Germain-l'Auxerrois, Marais en avait été « chassé » parce que, adolescent, sa voix avait mué. Comme le Je s'« ensevelit » après sa mue dans la musique instrumentale, Marais lui aussi s'est donné entièrement à la musique. En jouant de la viole, ce musicien, «ambitionnait d'imiter "les plus beaux agréments de la voix humaine" » $(L M, 17)$. Mais, « chassé » du chœur, il ne put « atteindre la maîtrise de la voix humaine » que lui aurait permis le chant. Par une volonté et un effort de substitution, il aurait donc « cherché à atteindre la maîtrise de l'imitation de la voix humaine après qu'elle a mué » $(L M, 18)$. C'est la raison pour laquelle Marais apprit la viole, et le fit auprès de Sainte Colombe, le plus estimé et le plus grand des violistes du temps qui, selon un de ses biographes, était considéré comme celui qui s'était le plus approché de la «maîtrise de la voix humaine » par son jeu et ses compositions.

$8 \mathrm{Si}$, adolescent, Quignard a trouvé traumatique l'expérience de la mue, et si, dans La Leçon de musique, il la représente de façon similaire chez son personnage, c'est qu'elle est l'expérience d'une séparation définitive d'avec un état premier. La voix d'après la mue, «la voix masculine », est pour lui une « voix exilée de sa première terre » $(L M, 18)$. Et quand Quignard imagine Marin Marais travaillant sur sa viole à imiter «la basse de voix », c'est, dit-il, qu'il s'efforçait de "domestiquer la maladie sonore, de panser l'affection de la voix humaine masculine» $(L M, 18)$. Telle est donc pour lui la voix de 
l'homme adulte: la manifestation d'une "maladie sonore»; l'expression d'un exil fondamental. La mue est donc une expérience dont on ne se remet pas, mais qu'il faut gérer : c'est là une des fonctions de la musique. Il ne s'agit pas de jouer de la musique pour retrouver l'état premier de la voix avant la mue, mais bien d'«imiter» la voix d'après la mue. Cela veut dire que l'imitation de la voix humaine d'après la mue a pour objectif un apaisement du présent, une négociation avec lui.

Dans La Leçon de musique, il s'agit de « domestiquer la mue » - et, ce faisant, les effets de celle-ci $(L M, 19)$. Pour lui la musique est une façon de vivre le sentiment d'exil par rapport à l'enfance. Ainsi, se fondant sur un passage d'une lettre de Mozart à sa sœur Marianne, et d'une note de leur père, Quignard explique que Mozart adolescent rêve à la voix de Marianne, qu'« il nie la mue », et que pour lui, à cette époque, « composer de la musique, c'est recomposer un territoire sonore qui ne mue pas » $(L M, 36)$. Il y a une nostalgie pour une période antérieure à la mue chez Mozart adolescent. Quignard distingue cette attitude de Mozart de celle qu'il considère avoir été celle de Marais, dont la mue, «au contraire » d'un mouvement nostalgique, « le pousse à étreindre l'exil. C'est la basse de viole » $(L M, 36)$.

La conscience que l'imitation de la voix humaine par Marais est un moyen de panser la blessure ne veut pas dire que cela permet de comprendre ce qu'il cherche. Lecteur de Freud, Quignard propose d'abord une interprétation analytique. Se fondant « de façon scrupuleuse » sur un passage d'un biographe de Marais, il explique qu'au bout de six mois d'enseignement, estimant qu'il n'avait plus rien à enseigner à Marin Marais, Sainte Colombe cessa de le recevoir $(L M, 24)$. Néanmoins, pour « profiter encore du sçavoir de son Maître ", Marais se glissait sous « un petit cabinet de planches » que Sainte Colombe «avait pratiqué sur les branches d'un Mûrier afin d'y jouer plus tranquillement de la Viole » $(L M, 25)$.

11 Quignard se demande ce que signifie cette cloison de branches sous la cabane. La scène le frappe au point d'en faire la pierre angulaire de sa théorie des origines de la musique en l'homme : elle lui suggère «le secret de la musique » $(L M, 26)$. C'est un lecteur d'ouvrages psychanalytiques qui parle : « La cloison sonore est première dans l'ordre du temps. Mais je songe - avant que nous soyons enveloppés dans notre propre chair - à la cloison tégumentaire d'un ventre autre » $(L M, 26)$. Quignard pense au ventre maternel qui, avant la naissance, sépare du monde extérieur. Ventre, peau du ventre, «cloison » - c'est le mot qu'il emploie. Il pense également, dit-il, au vêtement que l'on porte, «cloison » lui aussi, en ce sens qu'il dérobe le sexe à «la curiosité d'autrui » $(L M, 26)$. Or, avance-t-il, «le secret de la musique » c'est « une espèce de son étouffé qui est comme le sexe dérobé » ( $L M, 26)$. C'est ce son «étouffé » en lui que le musicien chercherait à écouter et à faire écouter quand il compose ou joue de la musique.

"Oreille collée au bois, corps accroupi, le héros musicien répète une position plus ancienne. Cette scène était une grossesse, devient un enfantement » $(L M, 29)$ : il ne s'agit pas ici, sous cette cabine de planches, d'une scène de «mue vocale masculine » (LM, 29), reconnait-il. Mais - et là Quignard utilise un autre sens du mot mue, faisant allusion à d'autres transformations -, il y a " une mue du chanteur en violiste », et cela se passe précisément sous un mûrier. Il rapproche l'arbre, le mûrier, du verbe mûrir et du mot maturation (LM, 29), et interprète : «La maturation auditive devient mutation d'un corps lové comme jadis dans le ventre maternel, désormais sous l'emprise d'un instrument à voix basse et, à certains égards, rouge foncé comme ces fruits » (LM, 29-30). Plus loin, Quignard raconte que Marais lui-même «s'était fait aménager un cabinet de musique qui 
donnait sur le jardin. [...] "C'était une réplique de la cabane de Sainte-Colombe" » (LM, 42). Marais répète ainsi le lieu sous lequel il se cachait dans sa jeunesse pour écouter son maître. L'épisode de mue adolescente - et la façon dont Marais y supplée - permet de répéter une écoute du monde rappelant celle qu'il avait pu faire enfant à travers la cloison du ventre maternel.

Pourquoi ce rappel de ce stade de la vie prénatale? C'est que, dit Quignard, la mue pubertaire en répète une autre, plus ancienne en nous. La musique en fait, explique-t-il, est en nous avant même le langage puisque dans le développement humain elle le précède, et qu'il y a " préhistoire, archaïsme de la musique en nous » $(L M, 27)$. Dans le développement de l'embryon, l'ouïe précède la voix, et dans l'enfant nouveau-né «le gazouillis, le chantonnement, le cri, la voix sont venus sur nous avant la langue articulée et à peu près sensée " $(L M, 27)$. C'est pourquoi, dans «Un épisode de la vie de Marin Marais », on peut lire : "L'oreille humaine est préterrestre et elle est préatmosphérique » $(L M, 52)$; c'est-à-dire qu'elle est déjà là avant notre arrivée au monde : « Avant le souffle même, avant le cri qui le déclenche, deux oreilles baignent durant deux à trois saisons dans le sac de l'amnios, dans le résonateur d'un ventre » $(L M, 52)$. Encore fotus, l'homme est, en se développant, déjà à l'écoute du monde et Quignard peut donc dire que «toute perception sonore " $(L M, 52)$ est reconnaissance. C'est parce que le son constitue son premier rapport au monde, que l'enfant entame avant même sa naissance un processus de reconnaissance. Après, il apprendra à passer des sons à la parole : la langue deviendra alors sa "première mue"; à cause d'elle il abandonnera «le gazouillement, le chantonnement, le cri ». La mue de la puberté ne fait que répéter cette première mue ( $L M$, 27), et la musique selon Quignard se définit en fonction de cet état : répétition de mue, elle représente l'« organisation de cette reconnaissance » $(L M, 52)$.

C'est bien de la voix humaine masculine que Quignard parle pour avancer dans sa "spéculation », comme il l'appelle lui-même. Chez la femme, si la mue se manifeste, c'est surtout plus tard dans la vie, à la ménopause (LM, 33). C'est pourquoi les femmes "persistent et meurent dans le soprano "; " leur voix est un règne [...] un soleil qui ne meurt pas » $(L M, 34)$; elles se distinguent des hommes qui, perdant leur voix d'enfant, se définissent comme des "humains que la voix a quittés comme une mue». Seuls les hommes « composent avec la perte de la voix et ils composent avec le temps. Ce sont des compositeurs. La métamorphose du grave à l'aigu ne leur est pas possible. Du moins corporellement : elle est instrumentalement possible. Elle a nom la musique » (LM, 37-38). Pour l'homme, la musique est donc métamorphose d'une substitution. Ce que Quignard redit exactement ou presque dans La Haine de la musique (HM, 155).

Il s'agit, donc, pour les hommes, de composer avec la perte de la voix. Et avec le temps. La musique est une relation au temps. Or qu'est-ce que le temps? La définition des origines de la musique en nous, de sa perpétuation par l'effort pour négocier les séquelles de la perte de la voix originaire inscrit celui-ci dans le temps, c'est-à-dire dans la mortalité. Dans La Leçon de musique, Quignard considère que « la seule expérience que le temps nous donne de lui-même », c'est « l'attente » (LM, 55-56). L'une des fins de la musique est de permettre d'endurer l'attente. De l'enfant, Quignard dit qu'il est impatient, «ne sait pas endurer le délai ». Or, la musique justement a pour objet de permettre à l'homme « d'endurer le délai» (LM, 56). «À la plainte de l'enfance: "c'est long", la musique répondait : "je consens à la longueur du temps" » (LM, 68).

C'est parce que la musique est consentement au temps que, selon Quignard, elle a partie liée à la souffrance humaine. «La souffrance humaine résonne dans le temps et dans la 
voix masculine» $(L M, 60)$; elle est «mue de mue » $(L M, 60)$, transformation de mue ; imitation de voix muée, métamorphose approximative, transformation. «Toujours, dit Quignard, quelque chose déchire l'instant. Et le déchiré, c'est moi » $(L M, 60)$. Moi, c'est-àdire lui bien sûr, mais aussi, sous l'angle de la musique, tout compositeur, instrumentaliste, auditeur. Pour faire pendant à l'étreinte de l'exil due à la perte de la voie pré-mue par Marin Marais, Quignard affirme qu'il faut un «récit» $(L M, 60)$, une " mélodie » $(L M, 61)$ pour gérer ce déchirement. Récit et mélodie sont sur le même plan, car du point de vue de ce rapport au temps, la musique ne diffère pas de la littérature, ou de tout autre art. «Il faut non seulement le récit pour aborder ma vie, mais un héros pour assurer la narration, un moi pour dire je. Il me faut une mélodie pour apaiser l'éventration du temps par le temps » (LM, 60-61).

Toute souffrance, en criant vengeance, n'appelle pas autre chose dans l'univers qu'un récit. Une dévoration fictive. Ce sont toutes les religions. Il n'y a pas de dieu sans mouvement de mâchoire. Un dieu n'est que cela - à condition que ses mâchoires se referment et que le sang coule, et qu'une meute s'agglutine autour de cette trace. (LM, 63-64)

Le constat de la mue masculine amène à comprendre l'interrogation sur la musique, d'un point de vue qui montre que, au-delà de la musique, c'est la signification même de notre rapport au monde qui est en jeu. Composer et jouer de la musique, c'est écrire, faire de l'art, inventer récits et mélodies, et c'est vivre avec, faire avec cette cruauté comprise ici sous l'angle d'un modèle métaphysique théologique ; c'est vivre avec les lois du récit ou de la mélodie, c'est-à-dire, de l'ordre, de l'autorité, du pouvoir, des idéologies, religieuses ou autres, qui appellent le sang.

Quignard clôt sa spéculation sur la musique dans "Un épisode de la vie de Marin Marais ", en articulant trois allégories. La première part d'une définition de l'essence de la musique qu'il donne en se référant au meurtre de l'empereur Tibère. À la question: "Quelle est l'essence de la musique pour moi ? ", il répond : "c'est le bruit étouffé que fit Tibère mourant - étouffé entre deux matelas sous les mains de Macron» - le préfet romain qui le fit assassiner $(L M, 75)$.

Interrompant cette première allégorie, Quignard passe aux deux autres, sans lesquelles le sens qu'il donne à la première ne serait pas compris.

La deuxième est un détournement transgressif du récit de la venue des Mages :

Dieu est éternel. Il était enfant. Il était soprano. Il ne connaissait pas encore le langage. Il était Dieu. Il était dans une crèche. Ils arrivèrent. On les appelait les rois mages. Ils étaient trois. Ils offrirent au jeune dieu le passé pour regretter, en sorte qu'il souffrît, le futur pour désirer, en sorte qu'il souffrît, le présent pour être accablé de l'un et de l'autre, en sorte qu'il souffrît. $(L M, 75)$

Revenant à « l'épisode » de la vie de Marin Marais se cachant sous la cabane du mûrier de Sainte Colombe, la troisième allégorie prolonge enfin cette allégorie christique subvertie de la souffrance humaine, et l'explique par l'exemple de la mort de Tibère. L'on sort de l'interprétation analytique (cloison de planches, cloison du ventre maternel) pour une autre : la place de Marin Marais sous la cabane, c'était «la place recroquevillée d'un homme. Tel le dieu soprano dans le bois d'une crèche. Le plancher, la vieille cloison, le vêtement, la peau. Ce sont les matelas. C'est la place de l'empereur. L'âme dans les instruments à cordes retient les deux cloisons sonores de s'écraser l'une sur l'autre » (LM, 76). 

enfin la signification de la première: "Sur le premier matelas, Tibère se débattait et tremblait. Le second matelas appuyait sur la vieille pomme d'Adam impériale et étouffait le son de douleur. Cette voix était muée. Les mains de Macron rendaient presque lointain et doux et comme enfantin le hurlement de l'empereur mourant " (LM, 77). Quignard explique que le premier matelas définit le rythme; le second l'intensité sonore. Ce qu'exprime l'empereur définit la mélodie. Macron est le nom de l'interprète (LM, 77). Telle est, à ce niveau de sa spéculation, la source de la musique. L'allégorie le dit: il y a cruauté, tragédie, inhumanité à l'origine de la musique. Pour le dire en un mot qu'il développera dans La Haine de la musique, ce que celle-ci fait entendre c'est une « souffrance sonore» $(H M, 16)$.

Haine de la musique (1996) se compose de douze «traités ». Dès le premier, «Les Larmes de saint Pierre », le sujet de l'ensemble qu'ils forment est posé : "J'interroge les liens qu'entretient la musique avec la souffrance sonore » (LM, 16). Thème qui semblerait répétitif par rapport à ce qui en est dit dans La Leçon de musique, si d'autres notions n'apparaissaient, précisant l'expérience du Je quignardien dans la nomination de l'origine de la musique en nous. Pour expliquer la nature de cette "souffrance sonore ", Quignard part de deux constats et en intègre ensuite les conséquences à ses propos sur le ventre maternel et l'idée de « cloison » dans La Leçon de musique. le feraient des linges, une "nudité sonore", une "souffrance sonore» en l'homme, et que, "à l'aide de ces linges nous soustrayons à notre propre oreille quelques sons et quelques gémissements plus anciens " $(H M, 13)$; le deuxième renvoie à deux mythes grecs. Celui d'Orphée, poète et musicien, tué, émasculé et «mangé cru » au cours de bacchanales sous l'impulsion d'Érato, mousa de la mousikè (HM, 14). L'autre, d'un autre musicien, Marsyas qui, ayant conseillé à Athéna d'abandonner sa flûte - afin de la lui dérober -, fut lui aussi émasculé et écorché vif (HM, 16, 157-158). Quignard retient l'idée que la violence meurtrière, le cannibalisme, l'omophagie, le meurtre, la castration, le mal, dit la représentation grecque archaïque de l'origine de l'œuvre d'art. Il voit dans ces deux mythes la représentation de l'origine de la musique. Pour lui, mousikè et pavor, musique et terreur, sont aussi liées que « le sexe et le linge qui le revêt » (HM, 16). Or, qu'est-ce que ce linge sinon "ce qui panse une plaie qui s'épanche », «qui dissimule une nudité qui fait honte ", «qui enveloppe l'enfant quand il sort de la nuit maternelle et découvre sa voix, émettant son premier hurlement »? (HM, 17). Les linges, c'est ce qui enveloppe le cri, le cache. D'où la question : qu'est-ce que nous écoutons en écoutant de la musique?

Ce premier moment spéculatif mène Quignard, dans La Haine de la musique, à prolonger son interprétation de la mue, donnée dans La Leçon de musique : d'où sa remarque : sons, fredons, musique, disent l'ancien temps qu'il fait en nous (HM, 20). Et c'est ce qu'ils en disent, ce qu'en dit la musique, que Quignard cherche à faire ressortir. Dans ce but, il revient au thème de la "cloison sonore ", auquel la musique lui semble originairement liée.

Dans La Haine de la musique, il présente des exemples extraits des journaux intimes de Sei Shônagon - confidente d'une impératrice japonaise de l'an mille - et d'Apronenia Avitia matrone romaine du IV siècle, protagoniste des Tablettes de buis d'Apronenia Avitia. Sei Shônagon se disait émue par le fait d'" "entendre derrière la cloison résonner les baguettes qui s'entrechoquent", "entendre le bruit que fait en retombant l'anse du vase où on met le vin de riz", entendre "le bruit faible des voix à travers une cloison" » (HM, 
21-22). Quignard trouve dans ces notations quelques exemples du «thème de l'oreille tendue » qu'on retrouve dans bien des mythes, contes et légendes de tous les pays, et qui montrent que « la musique est liée de façon originaire au thème de la "cloison sonore" » ( $H M, 22)$. Poursuivant sur ce thème, il montre cette fois qu'il s'agit moins d'apaiser la blessure due à la perte de la voix originaire, que « de ressusciter la curiosité sonore », de «refonder l'alerte animale» d'avant le langage articulé (HM, 22). Cette "curiosité sonore », ces sons avant langage, doivent être le premier rapport « musical» au monde, la première perception d'un langage avant l'accès au langage articulé.

Apronenia, elle, se disait « violemment » affectée par le " "bruit passionnant du cornet à dés" » (HM, 22-23). Ces deux mots, " passionnant » et « violemment », ont grande valeur pour Quignard, et lui permettent d'avancer l'idée qu'«il existe des bruits qui se sont "passionnés" en chacun d'entre nous » (HM, 22-23), des « bruits » qui disent, contiennent, les traces d'un " ancien temps", " il y a de même dans toute musique préférée un peu de son ancien ajouté à la musique même. Une mousikè au sens grec [...]» (HM, 23). Cela montre que la musique est originairement liée à la terreur. Dans la musique que nous écoutons, il y aurait aussi de la "musique ajoutée ", laquelle serait faite de terreurs anciennes, de persécutions que nous avons entendues avant de naître. La musique contiendrait cette violence panique dont parlent les mythes grecs.

Freud faisait remonter le grand premier conflit à l'œdipe. Quignard, lui, remonte au prénatal et même plus loin. "La connaissance d'un monde sonore sans capacité d'expression en retour, sans capacité d'appréhension ou de rebondissement verbal, et même l'oreille de la langue dans laquelle nous allons naitre, ces connaissances nous précèdent de plusieurs mois. De deux à trois saisons " (HM, 27-28). Pour le dire sans ménagements, «cette connaissance d'un monde sonore » daterait du moment même de notre conception.

$\mathrm{Au}$ temps de La Leçon de musique, Quignard expliquait celle-ci en fonction d'un thème analytique traditionnel, et l'image du refuge sous la cabane pour écouter les sons se rapportait à l'existence intra-utérine. Dans La Haine de la musique, c'est des sons mêmes, écoutés avant de naître et de leur identification qu'il s'agit. Quignard s'inscrit en faux contre toute idée de l'état pré-natal conçu comme un lieu paradisiaque de protection contre le monde. Le ventre maternel est selon lui le lieu premier de l'association entre musique et terreur. Terreur et violence sont partout. Le terror, écrit-il « est tout le fond de mon cœur » $(H M, 48)$. Il ajoute : « Nous avons tendu une oreille qui était terrorisée par des signes inintelligibles sous la cloison d'un ventre de peau avant même que nos poumons fonctionnent et qu'ils permettent de hurler " $(H M, 48)$. Il y a lieu de se demander ce que peut entendre de si terrifiant un fœtus... d'autant plus qu'un peu plus loin, dans un autre fragment, on lit: «tout est couvert du sang lié au son» (HM, 49). On pourrait se croire loin de la spéculation quignardienne sur l'appréhension de la musique comme imitation de la voix humaine après la mue. Il y avait, par cette imitation-là, négociation avec la blessure, séquelle de la perte de la voix originaire. Maintenant la question est de savoir ce que disait cette voix originaire, cette musique déjà en nous, et de quoi parle Quignard quand, à propos du ventre maternel, il décrit un état de «Guet permanent d'irruption, d'arythmie, de guerre, de soulèvement sous la menace de la mort» (HM, 30), de "passivité devant l'intrusion dont rien ne protège » (HM, 30). Ce que la musique fait entendre, ce avec quoi elle négocie, c'est cette origine de sons, de sang, de terreur, de fureur. 

surgissants» (HM, 55), ensuite, des tarabusts (HM, 62). Les fredons, "Vieux chants. Cantiques. Ritournelles enfantines et conjuratrices. Berceuses ou comptines. Polkas ou valses. Chansons de société et refrains populaires» (HM, 55) qui surgissent « inopinément» en nous. Les identifier permet de situer l'examen des origines de la musique par Quignard dans le voisinage de la psychanalyse. Dans le voisinage, non dans la psychanalyse même. Il explique : «les mots forment châne dans le souffle. Les images forment rêve dans la nuit. Les sons aussi forment chaîne le long des jours. Nous faisons aussi l'objet d'une "narration sonore" qui n'a pas reçu dans notre langue une dénomination telle que "le rêve" » (HM, 55). Quignard rapproche et distingue par là, en même temps, rêves et sons. Les images des rêves permettent des interprétations psychanalytiques. Le questionnement de Quignard concerne ce qu'il appelle la « narration sonore » en fonction de laquelle il construit sa compréhension du monde.

31 Cette distinction me suggère un néologisme: l'écoute et l'attention aux fredons, relèveraient d'une musanalyse comme, sur un autre registre, l'examen des rêves relève de la psychanalyse. Le fredon serait une donnée essentielle de notre rapport au monde, et cela même par lequel nous ne réussissons pas à cerner ce qu'est vraiment ce rapport. D'une part «le fredon resurgissant de façon inopinée renseigne sur l'état dans lequel on est $[. .] ».(H M, 56)$. D'autre part, il peut y avoir une «irritation à ne pas reconnaitre le nom, le titre, qui permettraient de maîtriser le fredon resurgi », à « ignorer le nom de ce qui hante le son» $(H M, 57)$. On sent que l'on sait ce que l'on fredonne, sans savoir le nommer, comme parfois on sait le mot désignant telle chose, sans parvenir à le trouver pour le dire. C'est le sujet de Le Nom sur le bout de la langue. Le fredon c'est quelque chose comme le son remontant du plus profond de nous, mais comme retenu dans le secret, au cours du mouvement de remontée.

D'où l'attention portée aux bruits qui nous rattachent au plus profond de nous-mêmes. D'où l'attention de Sei Shônagon, Apronenia Avitia, Marin Marais ou Sainte Colombe, qui, tous sentent en eux ces bruits passionnés et violents. «[...] le bruit de la cuillère cogne contre l'assiette de faïence [...], l'arc musical remonte. L'émotion nous prend d'un seul coup. Tout bouleverse les rythmes du corps, mais rien qui ait réellement sens ne peut être fourni » (HM, 60-61). Si la psychanalyse tente de faire sens de la scène analysée, et parfois le nomme, ce que j'appelle la musanalyse reste en deçà de la nomination et ne l'achève pas. Elle dit une fascination de ce qui obsède et, par là, permet l'élaboration de la scène fantasmatique de l'écriture chez Quignard.

Pour dire le caractère harcelant de ces bruits, Quignard propose le mot « tarabust », plus précis que "fredon» et emprunté à l'ancien français (le français moderne le connaît aussi). Il «désigne ce groupe de sons asèmes qui toquent la pensée rationnelle à l'intérieur du crâne et éveillent ce faisant une mémoire non linguistique » (HM, 62). Ce « tarabustant sonore datant d'avant le langage » $(H M, 62)$ dit à la fois « ce qui rabâche » et «ce qui tambourine» (HM, 62-63), un «bruit de querelle», de "résonateurs » et de « tambours» $(H M, 63)$. Il dit aussi des « coïts humains vociférants » et des "percussions d'objets creux » $(H M, 63)$. Voilà ce qui tarabuste dans le son qui revient, dans le bruit de la cuillère... Quignard insiste :

L'obsession sonore ne parvient pas à départager dans ce qu'elle entend ce qu'elle ne cesse de vouloir entendre et ce qu'elle ne peut pas ne pas avoir entendu. Un bruit incompréhensible et qui rabâche. Un bruit dont on ne savait pas s'il était querelle ou tambourinement, halètements ou coups. Il était très rythmé. Nous venons de ce 
bruit. C'est notre semence [...]. Toute femme, tout homme, tout enfant reconnait aussitôt le tarabust. (HM, 63) choses que nous n'osons révéler à nous-mêmes dans le silence, même dans les rêves que nous faisons...» (HM, 65), et il distingue entre «les fantasmes» relevant de la psychanalyse, et « les structures sonores » relevant de ce que j'ai appelé musanalyse. Ces dernières sont « plus anciennes que ces terrificatio visuelles » que sont les fantasmes.

Les tarabusts sont les fantasmes pour ce qui concerne les rythmes et les sons. Comme l'audition précède la vision, comme la nuit précède le jour, les tarabusts précèdent les fantasmes [...]. C'est ainsi que les idées les plus étranges ont un but, les goûts les plus singuliers une source, les manies érotiques les plus surprenantes une ligne d'horizon irrésistibles, les paniques un point de fuite invariable. (HM, 65-66) autrui, de même ferait la musanalyse quignardienne. Que faisons-nous quand nous fredonnons, chantons, jouons, obéissant au rythme de la musique qui monte en nous? Nous écoutons sans y penser - comme nous ne pensons pas nécessairement notre œdipe à tout moment, sauf lorsque l'examen analytique le rappelle -, ce qui fut notre temps d'avant notre temps, quand avant même de venir au monde, nous écoutions sans comprendre ce que l'examen des mythes et des contes, et des contenus du langage et des étymologies, et des fredons et des tarabusts nous apprend à identifier comme une violence énigmatique qui nous terrifiait, notre monde à venir et déjà là. Et comme la psychanalyse peut devenir une éthique - si on la comprend comme point de départ d'une vérité admise sur l'homme, pour tenter de vivre avec ce qu'elle révèle de peu de beauté, 
et de beaucoup de curiosité perverse en nous, mais afin de mieux établir sur une base de lucidité cette fois et non pas d'idéal naïf nos relations avec autrui, de même la musanalyse, dans l'esprit de Quignard, et de sa recherche de la "curiosité sonore» initiale de l'homme. La reconnaissance de l'expérience originaire du tarabust, de la musique, porte aussi en elle une éthique possible. C'est le sujet de Boutès, un de ses derniers livres. Boutès est un Argonaute. Lui aussi, comme Ulysse, a voulu écouter les Sirènes, mais, contrairement à ce dernier, il se leva simplement de sa place à la rame, et sans la moindre hésitation, plongea dans l'océan pour aller écouter les Sirènes au lieu même de leur chant, lieu d'origine de celui-ci, qui est aussi le lieu de la mort qu'elles donnent. Dans la mythologie quignardienne, il y a l'écoute prudente, rusée et protégée d'Ulysse. Et il y a l'écoute de Boutès, assumée dans le risque de comprendre le pourquoi de l'écoute, l'assomption lucide de violence, de la mortalité qu'elle dit.

Dans La Haine de la musique, Quignard disait des fotus qu'ils étaient comme des Ulysse attachés au mât de leur navire... Mais l'existence intra-utérine finit un jour, et la vie commence. Il y a des crises, des mues. Il faut négocier, tenir. Autant le faire comme Boutès. En écoutant dans la musique ses origines, sans ruse ni prudence. C'est ce que font presque tous les personnages de Quignard. Tant les déprimés de Carus, qu'Apronenia, Sei, Yo Ma, Tchen'Lien, Marais, Sainte Colombe, M. Settler, Ann Hidden, Charles Chegnogne, malades, déprimés, veufs ou solitaires, écoutant les bruits du monde, composant ou jouant de la musique.

\section{NOTES}

1. Marin Marais, 1656-1728 ; Jean de Sainte Colombe, né vers 1640, mort vers 1700.

2. La Haine de la musique (1996), rééd. Gallimard, « Folio », n. 3008 (voir aussi la bibliographie à la fin de l'article). Dorénavant, je me réfèrerai à ce titre par les initiales $H M$, suivi du numéro de la page citée. De même pour deux autres des livres de Quignard dont je citerai des extraits : $L M, L a$ Leçon de musique, « Folio », n. 3767, et VS, Vie secrète, « Folio », n. 3292.

\section{INDEX}

Mots-clés : Quignard (Pascal), musique, fredon, tarabust, musanalyse 